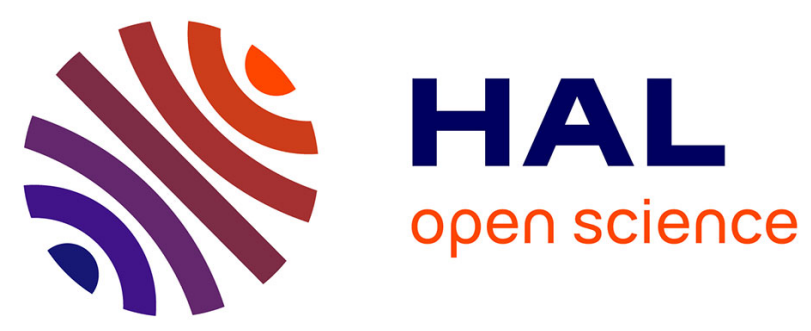

\title{
Soret-driven convection and separation of binary mixtures in a horizontal porous cavity submitted to cross heat fluxes
}

Loujaine Yacine, Abdelkader Mojtabi, Rachid Bennacer, Ali Khouzam

\section{To cite this version:}

Loujaine Yacine, Abdelkader Mojtabi, Rachid Bennacer, Ali Khouzam. Soret-driven convection and separation of binary mixtures in a horizontal porous cavity submitted to cross heat fluxes. International Journal of Thermal Sciences, 2016, 104, pp.29-38. 10.1016/j.ijthermalsci.2015.12.013 . hal01331777

\section{HAL Id: hal-01331777 \\ https://hal.science/hal-01331777}

Submitted on 14 Jun 2016

HAL is a multi-disciplinary open access archive for the deposit and dissemination of scientific research documents, whether they are published or not. The documents may come from teaching and research institutions in France or abroad, or from public or private research centers.
L'archive ouverte pluridisciplinaire HAL, est destinée au dépôt et à la diffusion de documents scientifiques de niveau recherche, publiés ou non, émanant des établissements d'enseignement et de recherche français ou étrangers, des laboratoires publics ou privés. 


\section{Open Archive TOULOUSE Archive Ouverte (OATAO)}

OATAO is an open access repository that collects the work of Toulouse researchers and makes it freely available over the web where possible.

This is an author-deposited version published in : http://oatao.univ-toulouse.fr/ Eprints ID : 15921

To link to this article : DOI:10.1016/j.ijthermalsci.2015.12.013 URL : http://dx.doi.org/10.1016/j.ijthermalsci.2015.12.013

To cite this version : Yacine, Loujaine and Mojtabi, Abdelkader and Bennacer, Rachid and Khouzam, Ali Soret-driven convection and separation of binary mixtures in a horizontal porous cavity submitted to cross heat fluxes. (2016) International Journal of Thermal Sciences, vol. 104. pp. 29-38. ISSN 1290-0729

Any correspondence concerning this service should be sent to the repository administrator: staff-oatao@,listes-diff.inp-toulouse.fr 


\title{
Soret-driven convection and separation of binary mixtures in a horizontal porous cavity submitted to cross heat fluxes
}

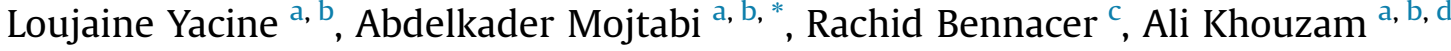 \\ a Université de Toulouse, INPT, UPS, IMFT (Institut de Mécanique des Fluides de Toulouse), Allée Camille Soula, F-31400 Toulouse, France \\ ${ }^{\mathrm{b}}$ CNRS, IMFT, F-31400 Toulouse, France \\ ${ }^{c}$ LMT /ENS-Cachan/CNRS/Université Paris Saclay, 61 avenue du Président Wilson, 94235 Cachan, France \\ ${ }^{\mathrm{d}}$ Arts et Métiers ParisTech, 2 bd du Ronceray, Angers, France
}

Keywords:

Convection

Soret effect

Porous medium

Species separation

Linear stability

\begin{abstract}
A B S T R A C T
An analytical and numerical study of Soret-driven convection in a horizontal porous layer saturated by a binary fluid and subjected to uniform cross heat fluxes is presented. The flow is driven by the combined buoyancy effect due to temperature and induced mass fraction variations through a binary water ethanol mixture. In the first part of the study, a closed-form analytical solution in the limit of a large aspect ratio of the cell $(A>>1)$ is developed. We are mainly concerned with the determination of the mass fraction gradient of the component of interest along the horizontal direction, which determines the species separation. In the second part, numerous numerical simulations are carried out in order to validate the analytical results and extend heat and mass transfer to an area not covered by the analytical study. Good agreement is found between analytical and numerical results concerning the species separation obtained for a unicellular flow. In this configuration, the Soret separation process is improved by two control parameters: the heat flux density imposed on the horizontal walls of the cell and the ratio, $a$, of heat flux density imposed on vertical walls to that on horizontal walls. The influence of the heat flux density ratio, $a$, on the transient regime (relaxation time) is also investigated numerically. We observe that an increase in the parameter $a$ leads to a decrease in the relaxation time. Thus, for a cell heated from below without lateral heating, the onset of convection from the mechanical equilibrium state is analyzed. The linear stability analysis shows that the equilibrium solution loses its stability via a stationary bifurcation or a Hopf bifurcation depending on the separation ratio and the normalized porosity of the medium. The linear stability results are widely corroborated by direct 2D numerical simulations. The thresholds of various multicellular solutions are determined in terms of the governing parameters of the problem using nonlinear direct numerical simulations.
\end{abstract}

\section{Introduction}

The aim of this work is to study, analytically and numerically, the species separation of a binary fluid mixture saturating a horizontal porous cavity of large aspect-ratio. The Soret effect is taken into account. The convective motion is driven using constant cross fluxes of heat on the horizontal and vertical walls.

Thermodiffusion corresponds to the migration of the components in a gaseous mixture or aqueous solution under the temperature gradient. Thermodiffusion in fluids, or the Soret effect, was discovered by Ludwig and Soret [1]. This phenomenon implies

\footnotetext{
* Corresponding author. Université de Toulouse; INPT, UPS; IMFT (Institut de Mécanique des Fluides de Toulouse), Allée Camille Soula, F-31400 Toulouse, France.
}

that any fluctuation in temperature will induce a variation in the concentration of a binary mixture. The Soret effect is much larger than the Dufour effect in liquid binary mixtures [2].

The magnitude of the Soret effect is associated with the Soret coefficient, which is the ratio of the thermodiffusion coefficient to the mass diffusion coefficient. Numerous previous works have shown the importance of the Soret effect on the behavior of multicomponent systems submitted to a temperature gradient. The coupling between the heat and species molecular transports is described by the mass flux density vector:

$$
\boldsymbol{J}_{m}=-\rho D \nabla C-\rho C(1-C) D_{T} \nabla T
$$

The first term of this equation comes from Fick's law and the second term describes the Soret effect (thermodiffusion). $\nabla C$ is the 


\begin{tabular}{|c|c|c|c|}
\hline \multicolumn{2}{|c|}{ Nomenclature } & \multicolumn{2}{|c|}{ Greek symbols } \\
\hline & & $\beta_{C}$ & Solutal expansion coefficient \\
\hline$a$ & Heat flux density ratio & $\beta_{T}$ & Thermal expansion coefficient $\left[\mathrm{K}^{-1}\right]$ \\
\hline A & Aspect ratio & $\lambda_{f}^{\prime}$ & Fluid thermal conductivity $\left[\mathrm{W}(\mathrm{mK})^{-1}\right]$ \\
\hline K & Permeability of the porous medium & $\lambda^{t}$ & Effective porous thermal conductivity $\left[\mathrm{W}(\mathrm{mK})^{-1}\right]$ \\
\hline$k$ & wave number & $\mu$ & Dynamic viscosity [Pas] \\
\hline$B_{S}$ & Mass fraction gradient in the $\mathrm{x}$ direction & $\nu$ & Kinematic viscosity $\left[\mathrm{m}^{2} \mathrm{~s}^{-1}\right]$ \\
\hline$B_{T}$ & Temperature gradient in the $\mathrm{x}$ direction & $\rho$ & Density $\left[\mathrm{kgm}^{-3}\right]$ \\
\hline$C_{0}^{\prime}$ & Initial mass fraction & $\psi$ & Separation ratio \\
\hline$D$ & Mass diffusion Coefficient $\left[\mathrm{m}^{2} \mathrm{~s}^{-1}\right]$ & $\varepsilon^{*}$ & Porosity of the medium \\
\hline$D_{T}$ & Thermodiffusion coefficient $\left[\mathrm{m}^{2}(\mathrm{sK})^{-1}\right]$ & $\varepsilon$ & Normalized porosity \\
\hline$g$ & Gravitational acceleration $\left[\mathrm{ms}^{-2}\right]$ & $\alpha$ & Effective thermal diffusivity $\left[\mathrm{m}^{2} \mathrm{~s}^{-1}\right]$ \\
\hline $\mathrm{H}$ & Height of the enclosure $[\mathrm{m}]$ & $\gamma$ & Heat capacity ratio \\
\hline$J_{m}$ & Mass flux density vector $\left[\mathrm{kg}\left(\mathrm{m}^{2} \mathrm{~s}\right)^{-1}\right]$ & $\varphi$ & Stream function \\
\hline$L$ & Length of the enclosure $[\mathrm{m}]$ & $\varphi_{0}$ & Intensity of the velocity field \\
\hline Le & Lewis number & $\sigma$ & Temporal amplification of the perturbation \\
\hline$P$ & Pressure [Pa] & $\omega$ & Angular frequency \\
\hline$R a$ & Thermal Rayleigh number & & \\
\hline$S$ & Species separation & \multicolumn{2}{|c|}{ Superscript } \\
\hline$S_{T}$ & Soret parameter $\left[\mathrm{K}^{-1}\right]$ & , & For dimensional variables \\
\hline$t$ & Dimensionless time & 0 & Refers to a reference state \\
\hline$T$ & Dimensionless temperature & $\mathrm{cr}$ & Refers to a critical value \\
\hline$T_{0}^{\prime}$ & Reference temperature & & \\
\hline$(u, v)$ & Dimensionless velocity components in Ref. $(\mathrm{x}, \mathrm{z})$ & & \\
\hline
\end{tabular}

mass fraction gradient induced by the temperature gradient, $\nabla T$, [3]. Although, the assumption $C(1-C) \approx C_{0}\left(1-C_{0}\right)$, where $C_{0}$ is the initial value of the mass fraction, is widely used, it remains valid within the limits of very small variations of the mass fraction around $C_{0}$. This assumption does not take account of the variation of $D_{T}$ with respect to the mass fraction $C$, such as in water-ethanol systems (Kolonder et al. [4]), or the dependence on temperature, such as in water- $\mathrm{NaCl}$ close to $12{ }^{\circ} \mathrm{C}$ (Mojtabi et al. [5]). In the present work, $D_{T}$ is assumed to be constant.

Despite its very small values of $D_{T}$, the Soret effect induces significant mass fraction variation in many natural or technological processes. The coupling of convection and thermodiffusion is called thermo-gravitational diffusion. This phenomenon was pointed out about eighty years ago with the experimental work of Clausius and Dickel [6], and by theoretical work of Furry et al. [7], which opened the way for the study of thermo-gravitational diffusion in vertical columns (TGC). In 1959, Lorenz and Emery [8] suggested introducing a porous medium in the thermogravitational column to reduce the convective motion. Thus, in a column with a large space between the two plates, filled with a porous medium, the fluid velocity is of the same order as TGC with a small gap. Thus, many works related to species separation using thermogravitational diffusion have been conducted in vertical columns. So the resulting flow intensity is controlled by the porous media permeability and an alternative means of control may be accessed by reducing the gravity component.

In 2003, Platten et al. [9] showed that the separation of species could be enhanced when the column was tilted. They concluded that separation could considerably increased by choosing an optimal inclination of the column. Charrier-Mojtabi et al. [10] showed that it was possible to perform the separation in a horizontal configuration when the separation ratio was negative or greater than a positive value leading to the onset of unicellular flow. They also showed that the Rayleigh number leading to optimum separation in a horizontal cell was greater than that the one obtained in a vertical cell (TGC). This allowed species separation in a thick cell (Elhajjar et al. [11]). Bennacer et al. [12] used a partitioning technique in order to enhance the separation within a vertical annular porous cylinder. They reported that the separation increased when the cylindrical annulus curvature increased and showed that the separation ability increased with a porous layer partitioning due to cross flow resulting from the co-rotative cells. Khouzam et al. [13] presented a new configuration leading to species separation in a horizontal rectangular cavity, heated from above or from below, in the presence of mixed convection (lid driven cavity). Their study showed that the separation could be increased for an optimal coupling between the imposed horizontal flow velocity and the flow induced by temperature gradient.

In Section 2, we present the physical problem and the mathematical formulation. An analytical model, based on the parallelflow approximation for a cell with large aspect ratio, is proposed in Section 3. In Section 4, the maximum species separation is determined for optimal values of thermal Rayleigh number, $R a$, and heat flux densities ratio, $a$. Most of the results were obtained for water-ethanol mixtures. The numerical method (FEM) employed to solve the governing equations is also presented. In Section 5-1, an analytical and numerical stability analysis is performed in order to obtain the critical values associated with the onset of the unicellular convection in the particular case $(a=0)$, and either stationary or Hopf bifurcation is obtained. In section $5-2$, direct numerical simulations are used to analyze multicellular flows obtained after the onset of convection.

\section{Mathematical formulation}

The system under study consists of a horizontal rectangular porous cavity with large aspect ratio $A=L / H$, where $H$, is the height of the cavity along the $\mathrm{z}$-axis and $\mathrm{L}$ is its length along the $\mathrm{X}$-axis (Fig. 1). The reference frame is in the middle of the cavity. The cavity is filled with a binary fluid mixture of density $\rho$ and dynamic 
viscosity $\mu$. All four walls are assumed to be impermeable and submitted to uniform heat fluxes of density $\mathrm{q}_{1}$ on the long horizontal sides and $\mathrm{q}_{2}$ on the short vertical sides, opposite plates being heated and cooled.

The Boussinesq approximation is assumed to be valid, so the thermo-physical properties of the binary fluid are considered constant, except the density in the buoyancy term which varies linearly with the local temperature, $\mathrm{T}$, and mass fraction, $\mathrm{C}$, of the denser component.

$\rho^{\prime}=\rho_{0}^{\prime}\left[1-\beta_{T}\left(T^{\prime}-T_{0}^{\prime}\right)-\beta_{C}\left(C^{\prime}-C_{0}^{\prime}\right)\right]$

where $\beta_{T}$ and $\beta_{C}$ are, respectively, the thermal and mass expansion coefficients of the binary fluid coefficients defined by: $\beta_{T}=-\frac{1}{\rho_{0}}\left(\frac{\partial \rho^{\prime}}{\partial T^{\prime}}\right)^{\text {of }}>0, \beta_{C}=-\frac{1}{\rho_{0}}\left(\frac{\partial \rho^{\prime}}{\partial C^{\prime}}\right)^{2}<0$.

In the present work, we focus on the optimal species separation between the two horizontal ends of the cell, which appears after the unicellular flow is established for low values of the Rayleigh number and for positive separation ratio. The parameters of the system studied correspond to a realistic binary mixture (waterethanol) with the initial mass fraction of the heavier component (water) $\mathrm{C}_{0}=0.6088 \% \mathrm{wt}$. The kinematic viscosity and the density are: $\nu=2.716 \times 10^{-6} \mathrm{~m}^{2} \mathrm{~s}^{-1}, \rho=935.17 \mathrm{~kg} \mathrm{~m}^{-3}$. Other relevant properties are $D=4.32 \times 10^{-10} \mathrm{~m}^{2} \mathrm{~s}^{-1}, D_{T}=1.37 \times 10^{-12} \mathrm{~m}^{2} \mathrm{~s}^{-1} \mathrm{~K}^{-1}$, $\beta_{c}=-2.12 \times 10^{-1}, \beta_{T}=7.86 \times 10^{-4} K^{-1}$, extracted from Platten et al. [9]. Thermal diffusivity and conductivity are, $\alpha_{f}=0.8771 \times 10^{-7} \mathrm{~m}^{2} \mathrm{~s}^{-1}, \lambda_{f}^{\prime}=0.33794 \mathrm{Wm}^{-1} \mathrm{~K}^{-1}$, from Wang and Fiebig [14].

The heat and the mass flux in the binary mixture are coupled due to the Soret effect and both contribute to the density gradient. The buoyancy force $\rho \vec{g}\left(\vec{g}=-g \overrightarrow{e_{z}}\right)$ is thus influenced by the Soret effect, considering that the Dufour effect describing currents of heat driven by concentration gradients can be neglected. Under these conditions, the mathematical model governing this problem, which includes the conservation equations (mass, Darcy, energy and chemical species), are written respectively as follows:
Typically, in Soret-driven convection, if the variation of the mass fraction is small, $C^{\prime}\left(1-C^{\prime}\right)$ can be replaced by $C_{0}^{\prime}\left(1-C_{0}^{\prime}\right), C_{0}^{\prime}$ being the mass fraction in the initial state.

The reference scales for length and time are, $H^{2} \gamma / \alpha$ (where $\gamma=(\rho c)^{\prime} /(\rho c)_{f}$, where $(\rho c)^{\prime}$ and $(\rho c)_{f}$ are respectively the effective and fluid volumetric heat capacity), and for pressure and velocity are $\alpha \mu / K, \alpha / H$ with $\alpha=\lambda^{\prime} /(\rho c)_{f}\left(\alpha\right.$ and $\lambda^{\prime}$ are the effective thermal diffusivity and effective conductivity of the porous-mixture system). The dimensionless temperature and mass fraction are given by:

$T=\frac{T^{\prime}-T_{0}^{\prime}}{\Delta T^{\prime}}, \quad c=\frac{C^{\prime}-C_{0}^{\prime}}{\Delta C^{\prime}}$ with $\Delta T^{\prime}=\left(q_{1}+q_{2}\right) H / \lambda^{\prime}$,

$\Delta C^{\prime}=\Delta T^{\prime} C_{0}^{\prime}\left(1-C_{0}^{\prime}\right)\left(D_{T} / D\right)$.

Thus, after eliminating the pressure, the dimensionless mathematical formulation of the problem is given by:

$\left\{\begin{array}{l}\frac{\partial u}{\partial z}-\frac{\partial v}{\partial x}=-R a \frac{\partial}{\partial x}[T-\psi C] \\ \frac{\partial T}{\partial t}+(\vec{V} \cdot \nabla) T=\nabla^{2} T \\ \varepsilon \frac{\partial C}{\partial t}+(\vec{V} \cdot \nabla) C=\frac{1}{L e}\left(\nabla^{2} C+\nabla^{2} T\right)\end{array}\right.$

As usual the equation of continuity is satisfied by introducing the stream function, $\varphi$, according to: $u=\partial \varphi / \partial z$ and $v=-\partial \varphi / \partial x$.

$\left\{\begin{array}{l}\nabla^{2} \varphi=-R a \frac{\partial}{\partial x}[T-\psi C] \\ \frac{\partial T}{\partial t}+(\vec{V} \cdot \nabla) T=\nabla^{2} T \\ \varepsilon \frac{\partial C}{\partial t}+(\vec{V} \cdot \nabla) C=\frac{1}{L e}\left(\nabla^{2} C+\nabla^{2} T\right)\end{array}\right.$

The problem under consideration depends on six nondimensional parameters: the Lewis number, $L e=\alpha / D$, the thermal Rayleigh number, $R a=g H^{2} K \beta_{T}\left(q_{1}+q_{2}\right) /\left(v \alpha \lambda^{\prime}\right)$, the separation

$$
\left\{\begin{array}{l}
\nabla \cdot \vec{V}^{\prime}=0 \\
\nabla P^{\prime}=\rho \vec{g}-\frac{\mu}{K} \overrightarrow{V^{\prime}} \\
(\rho c)^{*} \frac{\partial T^{\prime}}{\partial t^{\prime}}+(\rho c)_{f} \overrightarrow{V^{\prime}} \nabla T^{\prime}=\nabla \cdot\left(\lambda^{\prime} \nabla T^{\prime}\right) \\
\rho\left(\varepsilon \frac{\partial C^{\prime}}{\partial t^{\prime}}+\overrightarrow{V^{\prime}} \nabla C^{\prime}\right)=\nabla \cdot\left[\rho D \nabla C^{\prime}+\rho C^{\prime}\left(1-C^{\prime}\right) D_{T} \nabla T^{\prime}\right)
\end{array}\right.
$$

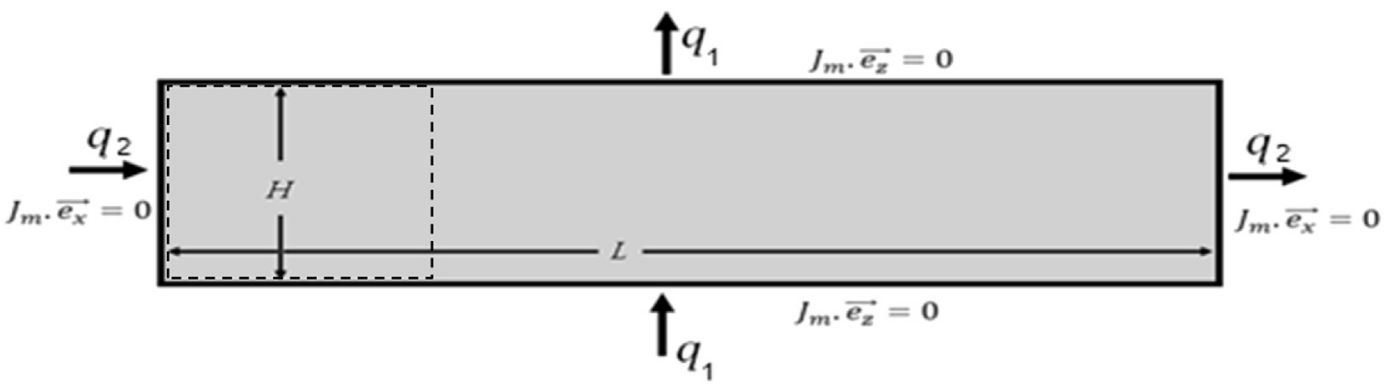

Fig. 1. Geometrical configuration of the cell submitted to cross heat fluxes. 
ratio, $\psi=-\frac{\beta_{C}}{\beta_{T}} \frac{D_{T}}{D} C_{0}^{\prime}\left(1-C_{0}^{\prime}\right)$, the normalized porosity $\varepsilon=\varepsilon^{*}(\rho \mathrm{c})_{\mathrm{f}} /$ $(\rho c)^{\prime}$, the horizontal to vertical heat flux densities ratio $a=q_{2} / q_{1}$ and the aspect ratio. $A=L / H$.

Uniform cross heat fluxes are applied on the four boundaries, and the walls are assumed to be impermeable with the slip condition on all walls,

$$
\left\{\begin{array}{l}
\forall z \in\left[-\frac{1}{2}, \frac{1}{2}\right] ; x= \pm \frac{A}{2}: \varphi=0 ; \frac{\partial^{2} \varphi}{\partial x^{2}}=0 ; \frac{\partial T}{\partial x}=-\frac{\partial C}{\partial x}=-\frac{a}{1+a} \\
\forall x \in\left[-\frac{A}{2}, \frac{A}{2}\right] ; z= \pm \frac{1}{2}: \varphi=0 ; \frac{\partial^{2} \varphi}{\partial z^{2}}=0 ; \frac{\partial T}{\partial z}=-\frac{\partial C}{\partial z}=-\frac{1}{1+a}
\end{array}\right.
$$

\section{Analytical solution}

For the present problem, the results are obtained within the limit of steady state and large aspect ratio $(A>>1)$, a regime where the parallel flow approximation is possible, which allows us to neglect the vertical velocity component (see, for instance, Bejan \& Tien [15]; Walker \& Homsy [16]), with the characterization of the temperature and concentration fields as linear stratification in the horizontal direction, after neglecting the side effects.

$\overrightarrow{V(z)}=u(z) \overrightarrow{e_{x}} ; \quad T(x, z)=B_{T} x+f(z) ; \quad C(x, z)=B_{S} x+g(z) ;$

With these assumptions (Eq. (7)) the system of dimensionless Eq. (5) with the boundary conditions (6) are reduced to a set of ordinary differential equations

$$
\left\{\begin{array}{l}
\frac{d^{2} \varphi}{d z^{2}}+R a\left[B_{T}-\psi B_{S}\right]=0 \\
B_{T} \frac{d \varphi}{d z}-\frac{d^{2} f(z)}{d z^{2}}=0 \\
B_{S} \frac{d \varphi}{d z}-\frac{1}{L e}\left(\frac{d^{2} g(z)}{d z^{2}}+\frac{d^{2} f(z)}{d z^{2}}\right)=0
\end{array}\right.
$$

Solving this system of Eq. (8) gives us the expression for the stream-function, the temperature and the mass fraction fields:

$$
\left\{\begin{array}{l}
\varphi(z)=\varphi_{0}(2 z-1)(2 z+1) \\
T(x, z)=B_{T} x+B_{T} \varphi_{0}\left(\frac{4 z^{3}}{3}-z\right)-\frac{z}{1+a} \\
C(x, z)=B_{S} x+\frac{\left(B_{S} L e-B_{T}\right) \varphi_{0}\left(4 z^{3}-3 z\right)}{3}+\frac{z}{(1+a)}
\end{array}\right.
$$

$\varphi_{0}$ represents the intensity of the velocity field. It indicates the maximum value of the stream factor, and is given by:

$\varphi_{0}=\frac{1}{8} R a\left[B_{S} \psi-B_{T}\right]$

The variations of the temperature and the concentration field determine the buoyancy force field which, in turn, drives the convective flow. In particular, the temperature gradients generate variations in the concentration field. On the other hand, concentration diffusion (Fick's law) and convective flow mixing tend to reduce these variations. The overall result of these changes is to close the feedback loop, which gives the possibility of species separation.

To determine the constants $B_{T}$ and $B_{S}$, a balance of heat and solutes through the sections of the rectangular cavity subdomain (see dashed line on Fig. 1) is established ([16-18]). Thus, by solving the dimensionless form of the mass conservation through the section:

$$
\int_{-0.5}^{0.5}\left(L e \cdot u(z) \cdot C(x, z)-B_{S}-B_{T}\right) d z=0 \quad \forall x \in[0, A]
$$

We have

$B_{S}=\frac{8 B_{T} \operatorname{Le} \varphi_{0}^{2}-15 B_{T}+10 \operatorname{Le} \varphi_{0} /(a+1)}{\left(8 L e^{2} \varphi_{0}^{2}+15\right)}$

and applying the condition on the thermal flow through sections (see dashed subdomain Fig. 1)

$$
\int_{-0.5}^{0.5}\left(u(z) \cdot T(x, z)-B_{T}\right) d z=-\frac{a}{1+a} \quad \forall x \in[0, A]
$$

gives the expression of $\mathrm{B}_{\mathrm{T}}$

$B_{T}=-\frac{5\left(2 \varphi_{0}-3 a\right)}{(a+1)\left(8 \varphi_{0}^{2}+15\right)}$

with $B_{S}$ defined as the mass fraction gradient along the $x$ axis. Thus, the species separation $S$ is defined as the difference in mass fraction of the denser species between the two ends of the domain considered, i.e. $x=0$ and $x=A$. Replacing $B_{T}$ and $B_{S}$, by their expression (Eqs. (12) and (14)), we deduce from Eq. (9) the equation giving the expression of $\varphi_{0}$ as a function of the controlling parameters $L e, \psi$ and $a$ :

$\varphi_{0}=\sum_{i=0}^{i=5} \alpha_{i} \varphi^{i}$

$$
\begin{aligned}
& \alpha_{0}=-225 \operatorname{Ra} a(\psi+1) \\
& \alpha_{1}=1800(a+1)-150 \operatorname{Ra}((\operatorname{Le}+1) \psi+1) \\
& \alpha_{2}=120 \mathrm{Le} \operatorname{Ra} a(\psi-L e) \\
& \alpha_{3}=960\left(\operatorname{Le}^{2}+1\right)(a+1)-80 L e^{2} \operatorname{Ra} \\
& \alpha_{4}=0 \\
& \alpha_{5}=512 \operatorname{Le}^{2}(a+1)
\end{aligned}
$$

This polynomial equation of the fifth degree admits five real or imaginary solutions depending on the values of the controlling parameters $R a, L e, \psi$ and $a$.

\section{Results and discussion}

Numerical simulations were performed using a commercial code (COMSOL Multiphysics) and were validated against the analytical results. Thus, the geometry was drawn, an aspect ratio $\mathrm{A}=10$ is considered. The flow domain was meshed using "quadrilateral" elements, the quadrangle spatial resolution is $30 \times 150$. A Lagrange-Quadratic scheme was selected to approximate the elements when discretizing the conservation Eq. (5) associated to the boundary conditions (6). The linear system solver "UMFPACK" was chosen for all our simulations. 
To determine the separation ability and compare the numerical to the analytical results (parallel flow), the curve $C=f(x)$ was plotted at a horizontal mid-plane ( $z=0$ for instance) and the slope was determined only in the central part of the cell, in order to eliminate the effects of recirculation in the vicinity of the vertical ends.

Examples of the variation of the iso-mass fraction and concentration field at different values of $R a$ and $a$ are presented in Figs. 2 and 3. The black lines represent the iso-mass fraction contour, while the colors represent the intensity of the concentration of the heaviest species.

From Fig. 2 (a), it can be seen that a very small value of $a$ (weak convective motion) results in almost horizontal and vertical concentration field stratification, and the iso-mass fraction contours are almost parallel to the horizontal walls. Thus the separation is small. The maximum of separation is obtained when the isoconcentration lines are inclined at around $145^{\circ}$ with respect to the horizontal Fig. 2 (b), which corresponds to the apparent optimal value of $a_{\mathrm{opt}}$. For values of $a$ exceeding $a_{\mathrm{opt}}$ the iso-mass fraction curvature increases and the separation decreases.

Likewise, from Fig. 3, we observe that the maximum separation takes place when iso-concentration lines are inclined at $145^{\circ}$, corresponding to optimal Rayleigh number $R a_{\text {opt. }}$. If the value of $R a$ is changed to greater than the optimal one, there is a deformation of the concentration field which leads to smaller separation, and for higher values of $R a$, the concentration field is significantly affected (as shown by the distortion of the iso-mass fraction lines in Fig. 3 (c)).
Fig. 4 shows the intensity of the flow in the point $(4,0)$ of the cavity, $\varphi_{0}$, as a function of $R a$ for $L e=203$ and $\psi=0.2$ (water/ ethanol mixture $60 \% / 40 \%$ ) and for two different values of the horizontal to vertical heat flux densities ratio, $a$. The four real analytical solutions of $\varphi_{0}$ are represented by solid and dashed lines and, these analytical results are corroborated by direct numerical simulations represented by points. As illustrated in Fig. 4, convection in the presence of side heating $(a \neq 0)$ is possible for any value of the Rayleigh number. This figure presents only the case of heating from the bottom $R a>0$. Analytical results show that it is possible to have either clockwise circulation of the flow $\varphi>$ 0or counterclockwise circulation $\varphi<0$. However, the side thermal effects tend to decrease the density in the left side of the cavity, which generates clockwise circulation $\varphi>0$ and means $\varphi_{0}<0$ in the center of the cavity since $\varphi=\varphi_{0}(2 z-1)(2 z+1)$.

As expected, the flow intensity is enhanced by the $R a$ value increase and the lateral heating, $a$, and the thermal solutal coupling (Soret separation) explains the intersection of the obtained curves. The numerical results fit the analytical curves well, validating the analytical approach restricted by the underlined assumptions.

The lateral heating enhances the flow intensity below a specific $R a$ value and more complex coupling occurs above this value. So to obtain the induced separation ability and the optimal cross heating conditions, we sought the optimal values.

Using the Maple software system, we determined the optimal value of $a=f(L e)$, and $R a=f(L e, \psi, a)$ leading to maximum of separation. Their expressions are as follows.

$$
\left\{\begin{array}{l}
a_{o p t}= \pm \frac{1}{12} \frac{\sqrt{30 L e}(\text { Le }-1)}{L e+1} \\
R a_{o p t}=\frac{120(1+a)^{2}}{( \pm 2 \xi+17 L e+12) a^{2} \psi+2( \pm \xi+6 L e+6) a^{2}+2( \pm \xi+5 L e) a \psi \pm 2 \xi a+5 L e}
\end{array}\right.
$$

The previous two figures validate the aspect ratio used to fit with the parallel flow assumption and linear temperature and concentration profile in the $\mathrm{x}$-direction.
Introducing the function $\xi=\sqrt{6 \frac{\mathrm{Le}^{2}\left(11 \mathrm{a}^{2}+10 \mathrm{a}+5\right)+6(2 \mathrm{Le}+1) \mathrm{a}^{2}}{(1+a)^{2}}}$ the maximum of separation $\mathrm{B}_{\mathrm{Smax}}$, is deduced and given by:

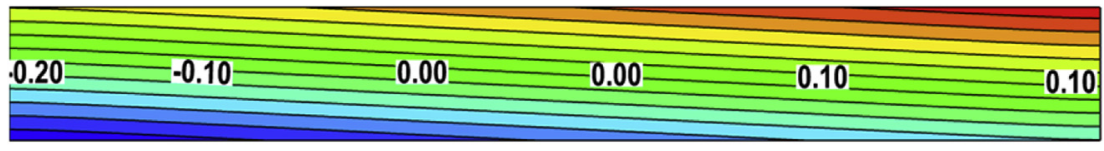

(a) $a=0.1$

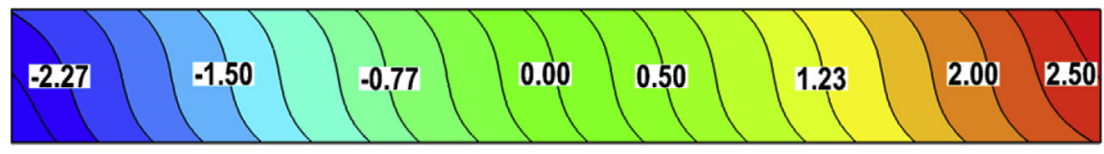

(b) $a=0.5$

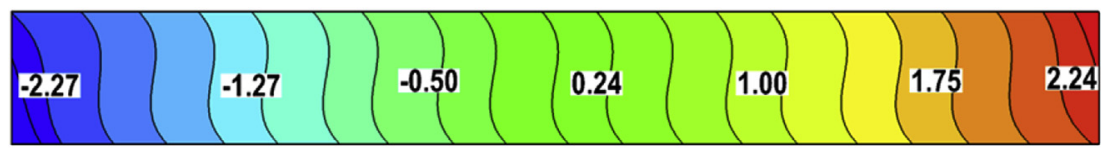

(c) $a=0.8$

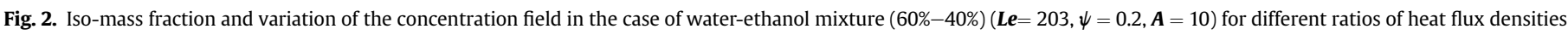
and for $\boldsymbol{R a}=0.1$. 


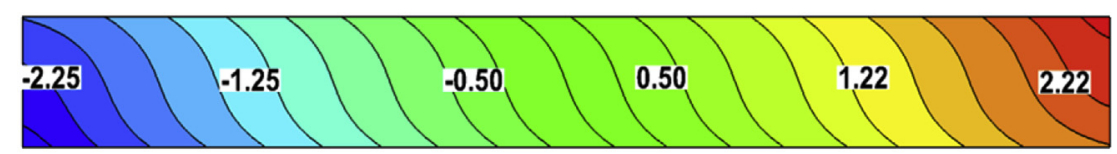

(b) $R a=0.2$

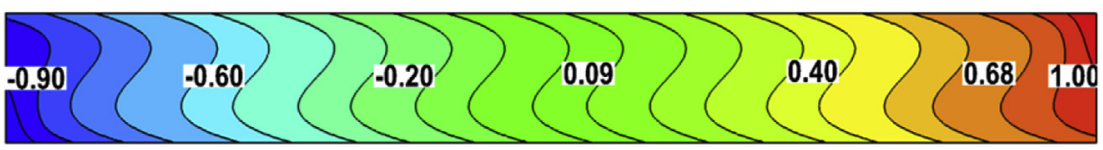

(b) $R a=1.0$

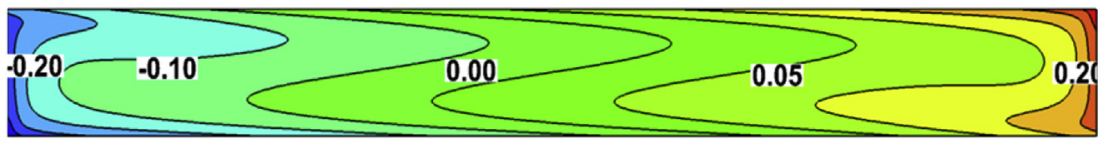

(c) $R a=5.0$

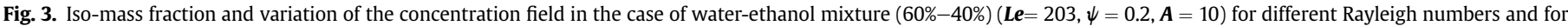
specific heat flux density $\boldsymbol{a}=0.2$

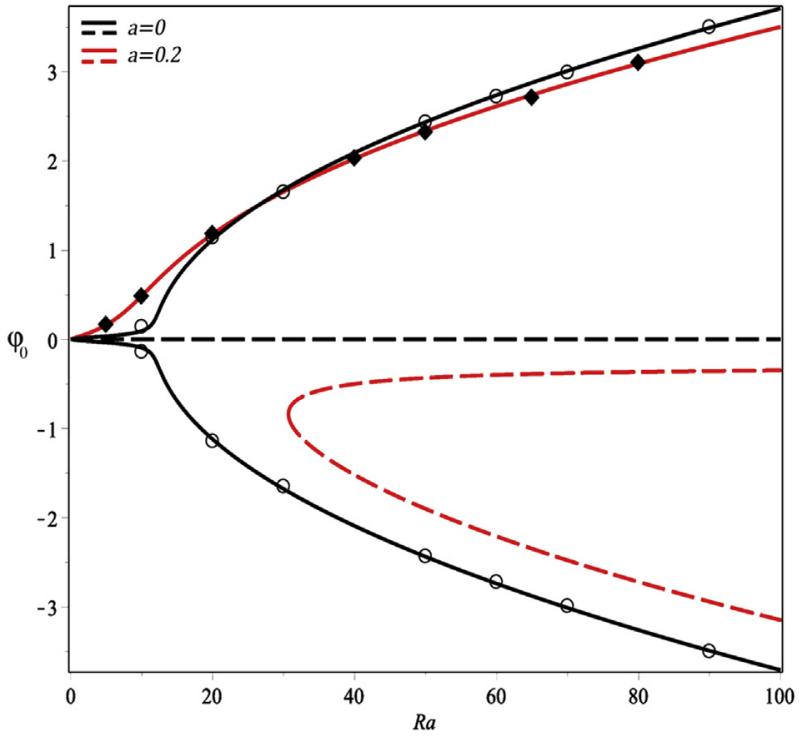

Fig. 4. The intensity of the flow $\varphi_{0}$ as a function of $\boldsymbol{R} \boldsymbol{a}$ for water-ethanol mixture and different values of $\boldsymbol{a}$, for $\boldsymbol{L} \boldsymbol{e}=203, \psi=0.2$.

$B_{\text {Smax }}=-\frac{1}{12} \frac{ \pm \xi(a+1)+6 a(L e-1)}{(a+1) L e}$

For the same conditions considered, Fig. 5 illustrates the variation of separation $B_{S}$ versus $a$ for various values of Rayleigh number $R a=0.1,0.5$ and 1 .

By increasing $a$, at fixed $R a$, we can see that the separation increases until it reaches a maximum value $S_{\max }$, $\left(B_{S \max }\right)$ for which optimal coupling between natural convection and thermodiffusion time is achieved. The optimal value of the heat flux densities ratio leading to $S_{\max }$ increases when $R a$ is decreased. When $a<a_{o p t}$ the thermodiffusion is predominant, the separation, due mainly to thermodiffusion, is small. Conversely when $a>a_{\text {opt }}$ the convection regime increases and tends to reduce the species separation.

The analytical solution is in good agreement with the $2 D \mathrm{nu}$ merical results (represented by dots) for all the values presented. The maximum of separation $B_{S \max }=0.501$ for $R a=0.1$ is obtained at $a=0.47$. For higher $R a=0.5$, the weaker $B_{S \max }=0.459$ is

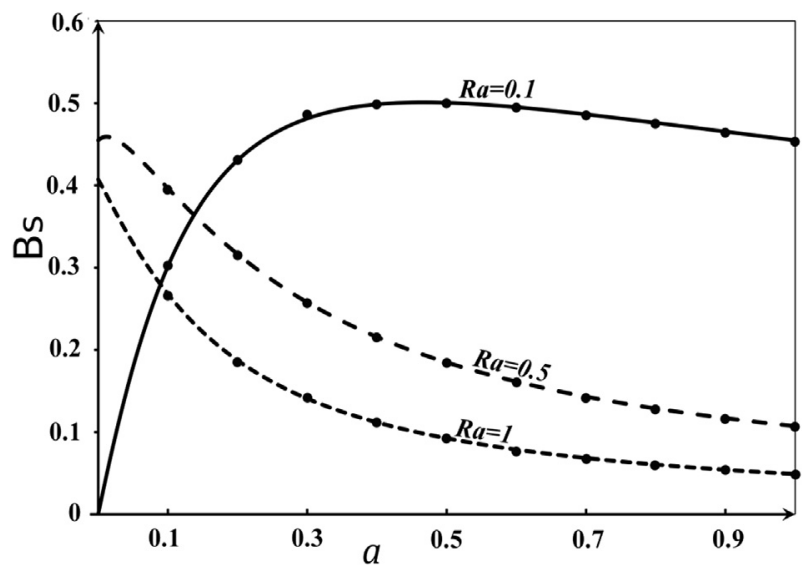

Fig. 5. Mass fraction gradient $\boldsymbol{B}_{\boldsymbol{S}}$ versus the flux ratio $\boldsymbol{a}$ for $\boldsymbol{R} \boldsymbol{a}=0.1,0.5$ and 1 (heated from below), $\boldsymbol{L} \boldsymbol{e}=203$ and $\psi=0.2$.

obtained at small $a=0.01$, and for $R a=1, B_{\operatorname{Smax}}=0.408$ is obtained at $a=0$.

Such optimal conditions are for a chosen case and the weak separation and optimal $R a$ are consequences of the weak mass diffusivity considered (Le 200). For such values, flow mixing is dominant.

The results presented above are for the thermally unstable case where the heating is from the bottom. The case of heating from the top is thermally stable but the induced separation can be stabilizing or destabilizing depending on the value of $\psi$. We will continue by focusing on positive $\psi$ and the evolution of separation with large Rayleigh number values is presented on Fig. 6. As before, a maximum of separation is found for an optimal value of $R a$, where optimal coupling between convection and thermodiffusion time is achieved. When the absolute value of the Rayleigh number is lower than the optimal value, thermodiffusion is predominant. In this case, the separation is small. Conversely, when the absolute value of $R a>R a_{\text {opt }}$, the convection regime increases and tends to reduce the species separation for both stabilizing and destabilizing thermal cases.

On the Fig. 6, we observe that as $a$ decreases, the location of the maximum separation is shifted towards higher absolute values of 


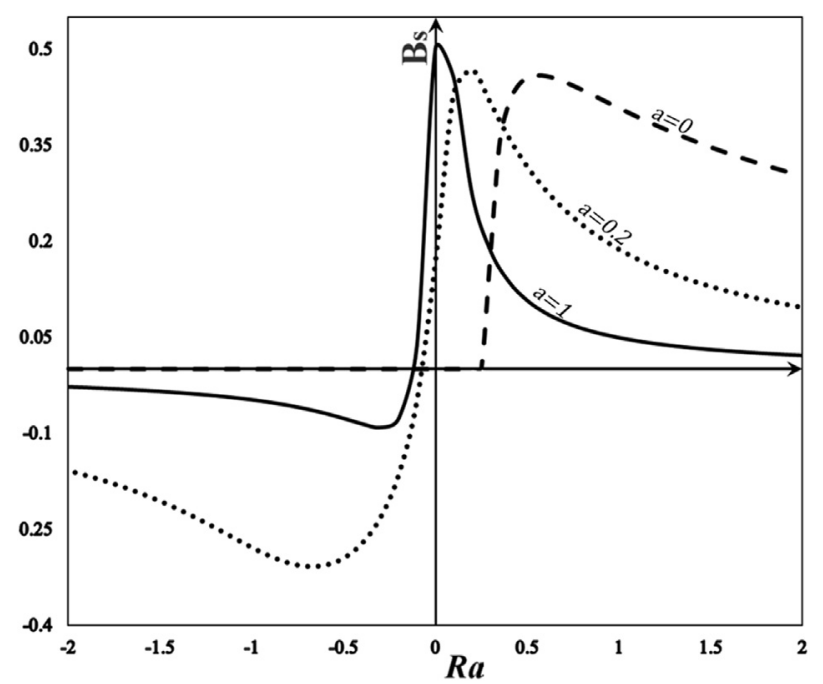

Fig. 6. Mass fraction gradient $\boldsymbol{B}_{\boldsymbol{S}}$ versus the Rayleigh number $\boldsymbol{R} \boldsymbol{a}$ for $\boldsymbol{a}=0,0.2$ and 1 , with $=0.2, \boldsymbol{L} \boldsymbol{e}=203$.

$R a$ but the global coupling shows a non-symmetrical tendency for the two cases.

In fact, with heating from below $(a>0$ and $R a>0)$ the heaviest component moves to the cold wall (the upper wall), which is an unstable situation. In this case, a small lateral heat flux density is enough to create motion in the mixture and reach the maximum of separation. On the other hand, when heating is from above ( $a>0$ and $R a<0)$, the heaviest component moves to the bottom wall (the cold one), which is a stable situation. In this case, a higher lateral heat flux density is required to obtain the optimal coupling between convection and thermodiffusion time which leads to the maximum of separation.

Here, we find the maximum of separation equal to $B_{\text {Smax }}=0.5878$ for $a=1$ and $R a=0.03$. In the other cases $B_{\text {Smax }}=0.474$ for $a=0.2$ and $R a=0.17$ and $B_{\text {Smax }}=0.459$ for $a=0$ and $R a=0.56$. Actually, having the maximum of separation at a greater value of $R a$ increases the time of separation and consequently the quantity separated.

The different optimal situations were analyzed in the steady state and the time needed to achieve such separation was at least as important as the separation level. Fast separation conditions could be interesting in multistage separation processes.

The separation process relaxation time (which is the time required to reach the steady state), was analyzed numerically for the products and mixture proportions (water-ethanol $60 \%-40 \%$ ) considered previously. The evolution of the mass fraction of the denser component as a function of time, for $R a=0.1$ and for various lateral heating, $a$, is illustrated in Fig. 7.

As we see from Fig. 7, the steady state can be reached considerably faster with high values of $a$, the critical times needed for $a=0.1,0.5$ and 1.0 are 1200, 650 and 450 respectively. A possible explanation for this would be that, the increase in the value of $a$ furthers the diffusion process and the flow intensity helps to reach the steady state in less time. The maximum separation is not monotonous with $a$ and exhibits a better separation for $a=0.5$. Nevertheless the decrease in separation ability is compensated by the fast separation ability where the ratio between the needed time and maximum separation, $\mathrm{t}_{\mathrm{cr}} / \mathrm{C}_{\max }$ are 1045,418 and 338.

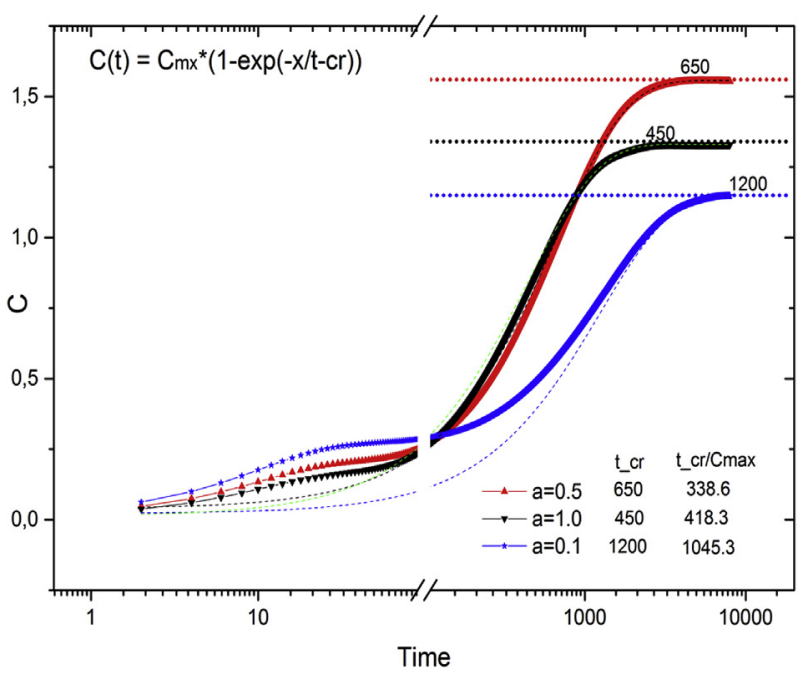

Fig. 7. The temporal evolution of the mass fraction, for different values of a and for $R a=0.1$.

\section{Linear and non-linear stability analysis for $a=0$}

\subsection{Convection in the particular case of $\boldsymbol{a}=0$}

The difference between the natural convection in laterally heated cavities and a cavity heated from below is well known theoretically and experimentally (Bejan [3]). In the absence of lateral heating, it is easy to show that there is a mechanical equilibrium solution characterized by:

$\left\{\begin{array}{c}\overrightarrow{V_{0}}=0 \\ T_{0}=-z \\ C_{0}=z\end{array}\right.$

In order to analyze the stability of this conductive solution, we introduce a vertical velocity component perturbation $w$, and perturbations of temperature, $\theta$, and concentration, $c$. If we assume that the perturbations $(w, \theta, c)$ are small, we obtain the following linearized equations

$\left\{\begin{array}{l}\nabla^{2} w=R a\left[\frac{\partial^{2}}{\partial x^{2}}(\theta-\psi c)\right] \\ \frac{\partial \theta}{\partial t}+w \frac{\partial T_{0}}{\partial z}=\nabla^{2} \theta \\ \varepsilon \frac{\partial c}{\partial t}+w \frac{\partial C_{0}}{\partial z}=\frac{1}{L e} \nabla^{2}(\theta+c)\end{array}\right.$

associated with the boundary conditions:

$w=0, \quad \frac{\partial \theta}{\partial z}=0, \quad \frac{\partial c}{\partial z}=0, \quad$ for $z=-\frac{1}{2}, \frac{1}{2} \forall x$

The perturbation quantities are chosen as follows:

$(w, \theta, c)=[\widehat{w}(z), \widehat{\theta}(z), \widehat{c}(z)] e^{i k_{x}+\sigma t}$

where $k$ is the wave number in the horizontal direction (ox) and $i^{2}=-1$, and $\sigma$ is the temporal amplification of the perturbation.

We developed two procedures to obtain the critical values of $\left(R a_{c s}, k_{c s}\right)$ and $\left(R a_{c o}, k_{c o, c o}\right)$. The first procedure was to solving the dispersion equation analytically for a stationary transition so as to obtain the exact values of the critical parameters numerically and 
the second one using the Galerkin method (long-wave and oscillatory cases) leading to approximate values.

\section{a Stationary transition}

In the first part, we focus on steady bifurcation. Eq. (19) with the boundary conditions 20 imply:

$\widehat{c}=-\widehat{\theta}($ Le +1$)+$ constant

This result shows, that, near the bifurcation point, the perturbed parts of the temperature and the concentration are similar, a result which is in a good agreement with our numerical simulations.

Eliminating $\mathrm{c}$ in the system of Eq. (19), we obtain a fourth-order system of equations governing the variables $w$ and $\widehat{\theta}$ :

$$
\begin{aligned}
& \left(D^{2}-k^{2}\right)^{2} \widehat{\theta}(z)+\operatorname{Rak}^{2}(1+\psi(1+L e)) \widehat{\theta}(z) \\
& \quad=\operatorname{Rak}^{2} \psi\left[\alpha_{1} \sinh (k z)+\alpha_{2} \cosh (k z)\right]
\end{aligned}
$$

where $D=d / d z$ and $\alpha_{1}$ and $\alpha_{2}$ are integration constants verifying

$\widehat{\theta^{\prime}}=0$ for $z=-\frac{1}{2}, \frac{1}{2} \forall x$,

$\widehat{\theta^{\prime \prime}}-k^{2} \widehat{\theta}=0$ for $z=-\frac{1}{2}, \frac{1}{2} \forall x$,

The characteristic equation associated with Eq. (23) is:

$$
\left(r^{2}-k^{2}\right)^{2}=\operatorname{Rak}^{2}(1+\psi(1+L e))
$$

The general solution of the fourth order ordinary differential equation (Eq. (23)) is given as a combination of four particular independent functions whose expression depends on the sign of. $R a(1+\psi($ Le +1$))$.

The solution obtained depends on four arbitrary constants. When we assume that this general solution verifies the boundary conditions (Eq. (20)), we obtain a homogeneous linear algebraic system with four equations and four unknowns corresponding to the four constants. This system has a nontrivial solution if the associated matrix determinant, $\operatorname{det}(R a, k, L e, \psi)$ is equal to zero. The expression of this determinant was obtained using the Maple algebra code.

Once we have calculated the determinant, we obtain the relation between the Rayleigh number, the wave number, the Lewis number and the separation ratio $\psi$ :

$$
\begin{aligned}
& 2 \sinh \left(R_{1}\right) \sin \left(R_{2}\right) k^{2}+2 \cosh \left(R_{1}\right) R_{1} R_{2} \cos \left(R_{2}\right)+\sinh \left(R_{1}^{2}\right) R_{1} R_{2} \\
& \quad-R_{1} \sin \left(R_{2}^{2}\right) R_{2}-R_{1} \cos \left(R_{2}^{2}\right) R_{2}-\cosh \left(R_{1}^{2}\right) R_{1} R_{2} \\
& \quad=0
\end{aligned}
$$

where

$$
\begin{aligned}
& R_{1}=\sqrt{K(k+\sqrt{R a(1+\psi(L e+1)})}, \\
& R_{2}=\sqrt{K(-k+\sqrt{R a(1+\psi(L e+1)})}
\end{aligned}
$$

We can then determine the exact value of the stationary critical Rayleigh number and the corresponding critical wave-number according to the separation ratio and the Lewis number (Table 1).
Table 1

Critical values of $R a_{c s}$ and $k_{c s}$ obtained with the exact solution and the Galerkin method, for the stationary bifurcations and for different values of $\psi$.

b Case of long-wave disturbances

\begin{tabular}{lllllll}
\hline & & \multicolumn{2}{l}{$L e=203$} & & \multicolumn{2}{l}{$L e=10$} \\
\cline { 3 - 4 } \cline { 5 - 6 } & & $R a_{c}$ & $k_{c}$ & & $R a_{c}$ & $k_{c}$ \\
\hline$\psi=-0.1$ & Exact & -0.619 & 0 & & -120.001 & 0 \\
& Galerkin & -0.619 & 0 & & -120.001 & 0 \\
$\psi=0$ & Exact & 12 & 0 & & 12 & 0 \\
& Galerkin & 12 & 0 & & 12 & 0 \\
& Exact & 0.561 & 0 & & 5.714 & 0 \\
& Galerkin & 0.561 & 0 & 5.714 & 0 \\
\hline
\end{tabular}

These results were confirmed using the Galerkin method for the perturbation quantities:

$$
\left\{\begin{array}{l}
\widehat{w}=\sum_{i=1}^{N} a_{[i]}\left(z-\frac{1}{2}\right)^{i} \cdot\left(z+\frac{1}{2}\right) \\
\widehat{\theta}=b_{[1]}+b_{[2]} \cdot z \cdot\left(z^{2}-\frac{3}{4}\right)+\sum_{i=1}^{N-2} b_{[i+2]}\left(z-\frac{1}{2}\right)^{i+1} \cdot\left(z+\frac{1}{2}\right)^{2}
\end{array}\right.
$$

Where $\widehat{w}(z), \widehat{\theta}(z)$ represent the vertical component of the velocity and temperature perturbation.

Table 1 reports the critical values of $R a_{c s}$ and $k_{c s}$ obtained with the exact solution and the Galerkin method, for the stationary bifurcations and for different values of. $\psi$ :

Although, in the general case, the solution of the eigenvalue problem (Eqs. (19) and (20)) can only be obtained numerically, the case of longwave disturbances (wave-number $k=0$ ) can be studied analytically (see, for instance, Charrier-Mojtabi et al. [10]). To study the behavior of long-wave disturbances, it is possible to develop the regular perturbation method with the wavenumber $k$ as a small parameter. In our case, using the Maple algebra code, we expand the determinant in the vicinity of $k=0$ to obtain

$$
\begin{aligned}
\operatorname{det}(\operatorname{Ra}(k), k, L e, \psi)= & \frac{R a}{1+\psi+\operatorname{Le} \psi}\left(f_{1}(R a, L e, \psi) k^{6}+f_{2}(R a, L e, \psi) k^{8}\right. \\
& +0\left(k^{10}\right)
\end{aligned}
$$

Setting the expression corresponding to the order 6 of the determinant development $\left(\mathrm{f}_{1}(R a, L e, \psi)\right)$ to zero, leads to

$R a_{c}=\frac{12}{1+\psi(L e+1)}$

In this case, we find that the line $\psi_{H}=-1 /(L e+1)$ is an asymptote of the curve. $R a_{\mathrm{cs}}(\psi)$.

\section{c. Oscillatory instability}

The linear stability Eq. (19) with the boundary conditions (20) are solved using the Galerkin method where the perturbations are chosen as Fourier functions and polynomial expansions. The convergence of the critical parameters obtained by these two approximations is similar. The purpose is to find an oscillatory instability $(\sigma=i \omega)$ for a Rayleigh number $R a_{c o}$ smaller than the one at which marginal stability $\sigma=0$ is observed. This stability study leads to a homogeneous linear algebraic system, which has a nontrivial solution if the associated matrix determinant is equal to zero. The Maple software was used for the symbolic calculations of the residue and of the $3 \mathrm{~N} \times 3 \mathrm{~N}$ determinant $\mathrm{A}$. 
With all the approximations used, the determinant has the following form:

$\operatorname{det}(A)=R(R a, \varepsilon, L e, \psi, k, \omega)+i S(R a, \varepsilon, L e, \psi, k, \omega)$, where $R$ and $S$ are real polynomial functions of $(R a, k, \omega, \varepsilon, L e, \psi)$. The degrees of the variables $R a$ and $k$ in these functions increase according to the order, $\mathrm{N}$, of truncation. We performed the symbolic calculations of the $3 \mathrm{~N} \times 3 \mathrm{~N}$ determinant $\mathrm{A}$ for levels $\mathrm{N}=3,4$ and 5 .

To calculate the value of the critical Rayleigh number corresponding to a Hopf bifurcation, we proceed as follows.

We first fix the particular values of $\psi, \varepsilon$ and Le. We resolve the following algebraic system, with two unknowns $(R a, \omega)$ and a parameter $k$ :

$\left\{\begin{array}{l}R(R a, k, \omega)=0 \\ S(R a, k, \omega)=0\end{array}\right.$

When we solve this system, we obtain the real roots of indices $\mathrm{j}$ : $R a_{j}=f_{j}(k)$ and $\omega_{j}=h_{j}(k)$. We can then look for the minimum value of Ra according to $\mathrm{k}$ and obtain the critical parameters $R a_{j c}=f_{j}\left(k_{c}\right)$ and $\omega_{j c}=h_{j}\left(k_{c}\right)$. In Fig. 8 (a) and (b), we present the stability diagrams

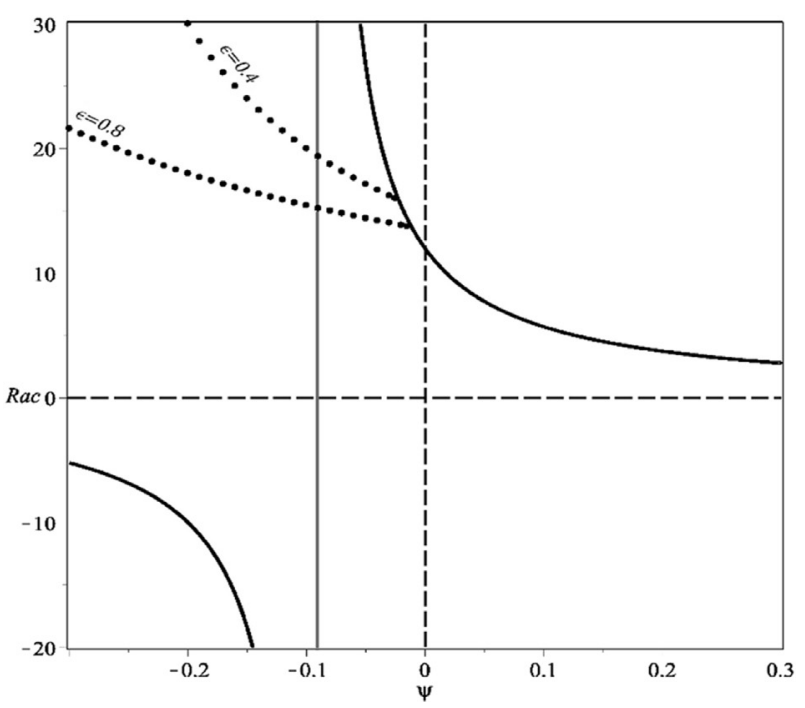

(a)

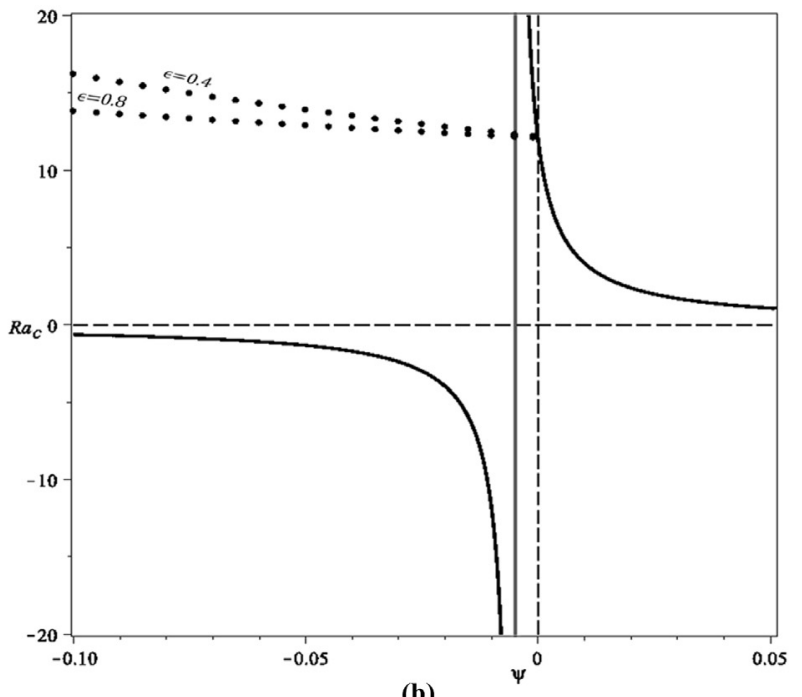

(b)

Fig. 8. Stability diagram of the equilibrium state for (a) $L e=10$, (b) $L e=200$ and for different porosity values $(\varepsilon=0.4,0.8)$. Solid lines: stationary bifurcation; dotted line: Hopf bifurcation.
$R a_{c}=f(\psi)$ obtained for $L e=10$ and 200 respectively (the solid lines are associated with the stationary bifurcation). Fig. 8a and b show that, for values of $\psi$ less than $\psi_{H}=-1 /(L e+1)$, the primary transition is a Hopf bifurcation. In this domain, we can see the important role of porosity: when it decreases, the stability of the equilibrium solution is reinforced.

\subsection{Nonlinear numerical analysis of the multicellular flow for $\boldsymbol{a}=0$}

The numerical solution of the problem admits, in principle, an infinity of solutions, each of them associated with a given set of initial conditions. Depending on the initial conditions introduced in the computations, the stationary solution is characterized by Ref. [1-9] rolls. In Fig. 9, we present the flow intensity as a function of the Rayleigh number for all numerical solutions that may exist in this domain of Rayleigh number. We started the numerical procedure using pure conduction, as the initial condition. For high values of thermal Rayleigh number, we found a particular multicellular flow. We used the values of velocity; thermal and solutal fields obtained for this multicellular flow as a new initial condition and progressively decreased the value of $R a$ until the nature of the multicellular flow changed. These solutions remained stable with decreasing Rayleigh number until they lost this stability via an instationary bifurcation. The structure of the flow obtained after each bifurcation point is associated with a smaller number of rolls. We also note that the intensity of flow increases as the number of rolls decreases.

\section{Conclusion and perspectives}

In this work, analytical and numerical studies were performed to investigate the species separation in a binary fluid saturating a horizontal porous layer subjected to cross heat fluxes. The Darcy model and the Boussinesq approximation were used to solve the governing equations of the problem. In this configuration, the two main control parameters are the thermal Rayleigh number, $R a$, and the heat flux density, $a$, for given values of Lewis number, $L e$, and separation ratio, $\psi$. The heat flux imposed on the vertical walls in addition to that imposed on the horizontal walls allows more mass fraction horizontal gradient evolutions in comparison to the case of classical thermogravitational columns where only one temperature gradient induces convective heat and mass transfer.

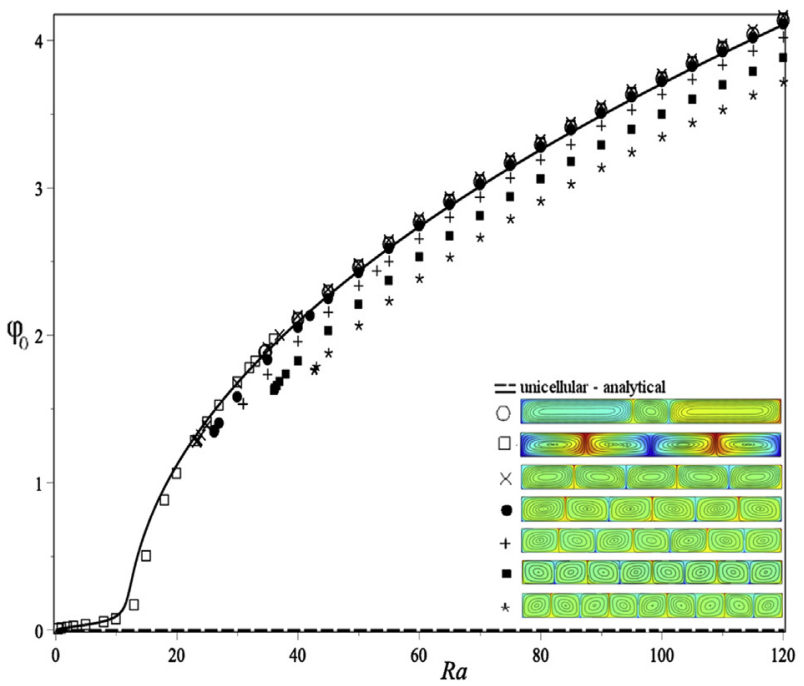

Fig. 9. The intensity of the flow $\varphi_{0}$ as a function of $\boldsymbol{R a}$ for water-ethanol mixture for all possible multicellular flows (symbols) compared with the unicellular flow (solid line). 
The results were obtained for a wide range of governing parameters, namely the thermal Rayleigh number, $R a$, and the flux ratio, $a$, for given values of the Lewis number, $L e$, and the separation ratio, $\psi$. In the first part of the paper, an analytical solution was determined in the case of a shallow cavity $(A>>1)$. This theoretical study allowed us to determine the expression for the velocity, temperature and mass fraction fields as a function of various dimensionless parameters of the problem. From this theoretical study, we deduced the optimal species separation values $\mathrm{B}_{\mathrm{Smax}}$ according to the two control parameters, $R a$ and $a$. The analytical results were corroborated by direct $2 \mathrm{D}$ numerical simulations using a commercial finite element code (COMSOL). The comparison with the separation obtained in other physical configurations showed that the species separation may be greater in this configuration even if the optimal separation values $B_{S \max }$ are equal in these different geometries.

All previous studies have mainly focused on the optimal species separation and avoided the transient regime to reach such a steady state. We observed that the real optimal situation for separation depends on a new criterion: the ratio of $t_{c r}$ to the maximal mass fraction gradient, $t_{\mathrm{cr}} / \mathrm{B}_{\mathrm{Smax}}$, where $\mathrm{t}_{\mathrm{cr}}$ is the time to reach the steady state. Such an optimal criterion needs to be completed in forthcoming studies and generalized versus the main control parameters, a and $R a$. For the particular case of no lateral flow $(a=0)$, the linear stability theory allowed us to predict the critical threshold of the convection according of the control parameters. The new possible multiple solutions depending on the initial state constitutes one of the interesting new challenges in the thermodiffusion area.

\section{References}

[1] C. Soret, Influence de la température sur la distribution des sels dans leurs solutions, 91, Compte-Rendu de l'Académie des Sciences, Paris, 1880, pp. $303-384$.
[2] J.K. Platten, M. Marcoux, A. Mojtabi, The Rayleigh-Benard problem in extremely confined geometries with and without the Soret effect, Comptes Rendus Mec. 335 (2007) 638-654.

[3] P. Costeseque, A. Mojtabi, J.K. Platten, Thermodiffusion phenomena, Comptes Rendus Mec. 339 (2011).

[4] P. Kolodner, H. Williams, C. Moe, Optical measurement of the Soret coefficient of ethanol/water solutions, J. Chem. Phys. 88 (1988) 6512-6524.

[5] A. Mojtabi, J.K. Platten, M.C. Charrier-Mojtabi, Onset of free convection in solutions with variable Soret coefficients, J. Non-Equilib. Thermodyn. 27 (2002) 25-44.

[6] K. Clusius, G. Dickel, New process for separation of Gas mixtures and isotopes Naturwissenschaften 26 (1938) 33.

[7] W.H. Furry, R.C. Jones, L. Onsager, On the theory of isotope separation by Thermal diffusion, Phys. Rev. 55 (1939) 1083-1095.

[8] M. Lorenz, A.H. Emery, The packed thermal diffusion column, Chem. Eng. Sci. 11 (1959) 16-23.

[9] J.K. Platten, M.M. Bou-Ali, J.F. Dutrieux, Enhanced molecular separation in inclined thermogravitational columns, J. Phys. Chem. B 107 (2003) $11763-11767$.

[10] M.C. Charrier-Mojtabi, B. Elhajjar, A. Mojtabi, Analytical and numerical stability analysis of Soret-driven convection in a horizontal porous layer, Phys. Fluids 19 (2007).

[11] B. Elhajjar, M.C. Charrier-Mojtabi, A. Mojtabi, Separation of a binary fluid mixture in a porous horizontal cavity, Phys. Rev. E 77 (2008) 6.

[12] R. Bennacer, A.A. Mohamad, M. El Ganaoui, Thermodiffusion in porous media: multi-domain constitutant separation, Int. J. Heat Mass Transf. 52 (2009) $1725-1733$.

[13] A. Khouzam, A. Mojtabi, M.-C. Charrier-Mojtabi, B. Ouattara, Species separation of a binary mixture in the presence of mixed convection, Int. J. Therm. Sci. 73 (2013) 18-27.

[14] J. Wang, M. Fiebig, Measurement of the thermal-diffusivity of aqueoussolutions of alcohols by a laser-induced thermal grating technique, Int. J. Thermophys. 16 (1995) 1353-1361.

[15] A. Bejan, C.L. Tien, Natural-convection in a horizontal porous-medium subjected to an end-to-end temperature difference, J. Heat Transfer-Trans. ASME 100 (1978) 191-198.

[16] K.L. Walker, G.M. Homsy, Convection in a porous cavity, J. Fluid Mech. 87 (1978) 449-474.

[17] O.V. Trevisan, A. Bejan, Mass and heat transfer by natural convection in a vertical slot filled with porous medium, Int. J. Heat Mass Transf. 29 (1986) 12

[18] A. Mahidjiba, R. Bennacer, P. Vasseur, Effect of the boundary conditions on convection in a horizontal fluid layer with the Soret contribution, Acta Mech. 160 (2003) 161-177. 